\title{
Dose Rate Considerations for Semiconductor Electronics: Why Current Variations Enable Unique GaN-based Transmission Electron Microscopy
}

Petra Specht ${ }^{1}$, Ronny Kirste ${ }^{2}$, Zlatko Sitar $^{2}$, Travis Anderson ${ }^{3}$, Andrew Koehler ${ }^{3}$ and Christian Kisielowski $^{4}$

${ }^{1}$ University of California in Berkeley, Berkeley, California, United States, ${ }^{2}$ NCSU, Raleigh, North Carolina, United States, ${ }^{3}$ NRL, Washington, District of Columbia, United States, ${ }^{4}$ The Molecular Foundry and Joint Center for Artificial Photosynthesis, Berkeley, California, United States

Recently, it was found that electron-beam induced material changes can be significantly suppressed when electron dose rates are applied, utilizing specific, low electron supply for structural analysis and / or time resolution [1-3] in transmission electron microscopy (TEM). Such techniques can be beneficial for the investigation of a large variety of beam-sensitive materials, from biological materials to catalytic nanoclusters.

In wide-bandgap semiconductor materials it is unusual to perform TEM using low-dose rate microscopy. $\mathrm{GaN}$ and its alloys in particular are electron-hard, which is understood as its device structures exhibiting no significant change of their respective performance parameters after pronounced electron beam exposure. The underlying theory for this behavior is based on high displacement energy, for both gallium and nitrogen atoms. This definition, however, is not necessarily applicable to thin TEM samples, in particular not to locally defective areas, and it does not take into account any material changes which do not displace atoms. We recently demonstrated that in $\mathrm{Mg}$-implanted $\mathrm{GaN}$ electron-beam induced changes occur at dose rates higher than $200 \mathrm{e}^{-}\left(\AA^{2} \mathrm{~s}\right)^{-1}$ [4], locally producing super-lattice structures. Similar changes were also found in nominally undoped, highly strained GaN which shows that locally the structure of GaN-based multi-layers can change significantly in a TEM.

A critical parameter for electronic device performance is the distribution of substitutional impurities (alloying). In order to ensure that the in-grown alloy distribution is not changed by the electron beam in a TEM analysis low dose rates were used for the investigation of various strained $\mathrm{Al}_{\mathrm{x}} \mathrm{Ga} 1-\mathrm{x} \mathrm{N}$ interface structures. It is to date still unclear if alloying $\mathrm{GaN}$ will result in a Poisson-distributed impurity coordination, one example of inhomogeneous distribution is shown in Fig. 2. TEM images were taken at the Molecular Foundry of LBNL [5], in the TEAM1 microscope (monochromated, aberration-corrected TEM), at 300kV. A Nelsonian illumination scheme was used and image series were recorded with dose rates lower than $200 \mathrm{e}^{-}\left(\AA^{2} \mathrm{~s}\right)^{-1}$ on a K2 camera (direct electron detector). As the observed epilayer structures were a few micrometers thick, FIB lamellae were rotated by 90 degrees and re-mounted prior to final thinning in a low voltage ion mill (Nanomill). Therefore, multiple interfaces could be investigated in atomic resolution on the same sample, see Figure 1. Using the low dose rate technique the question of impurity distribution in GaN-based structures can now be finally answered $[5,6]$. 


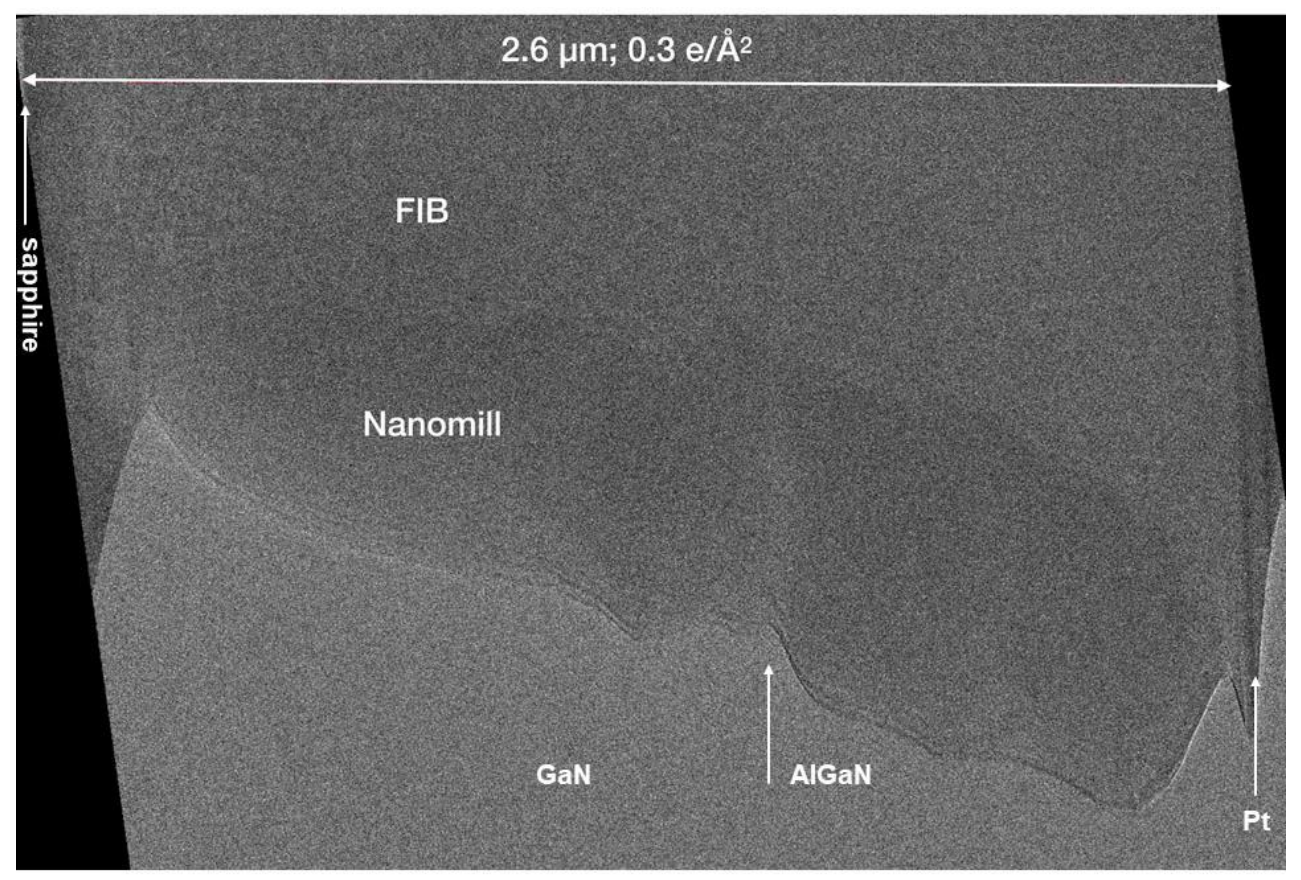

Figure 1. A typical multi-layer $\mathrm{Al}_{\mathrm{x}} \mathrm{Ga}_{1-\mathrm{x}} \mathrm{N} / \mathrm{GaN}$ device structure (here: Avalanche Photodiode) with epilayers of different materials. At the left the sapphire substrate is barely visible, at the right a Pt cover layer, from the FIB liftout, is shown. In between, the $2.6 \mu \mathrm{m}$ thick structure can be investigated at its whole thickness range, because the lamella was rotated so that it can be thinned along the sidewalls of the epilayer structure. Darker areas indicate where low voltage ion milling had occurred.

AIGaN Superlattices: Dose rate: 165 e $\AA^{-2} \mathrm{~s}^{-1} \quad$ Total dose: 9960 e $\AA^{-2}$

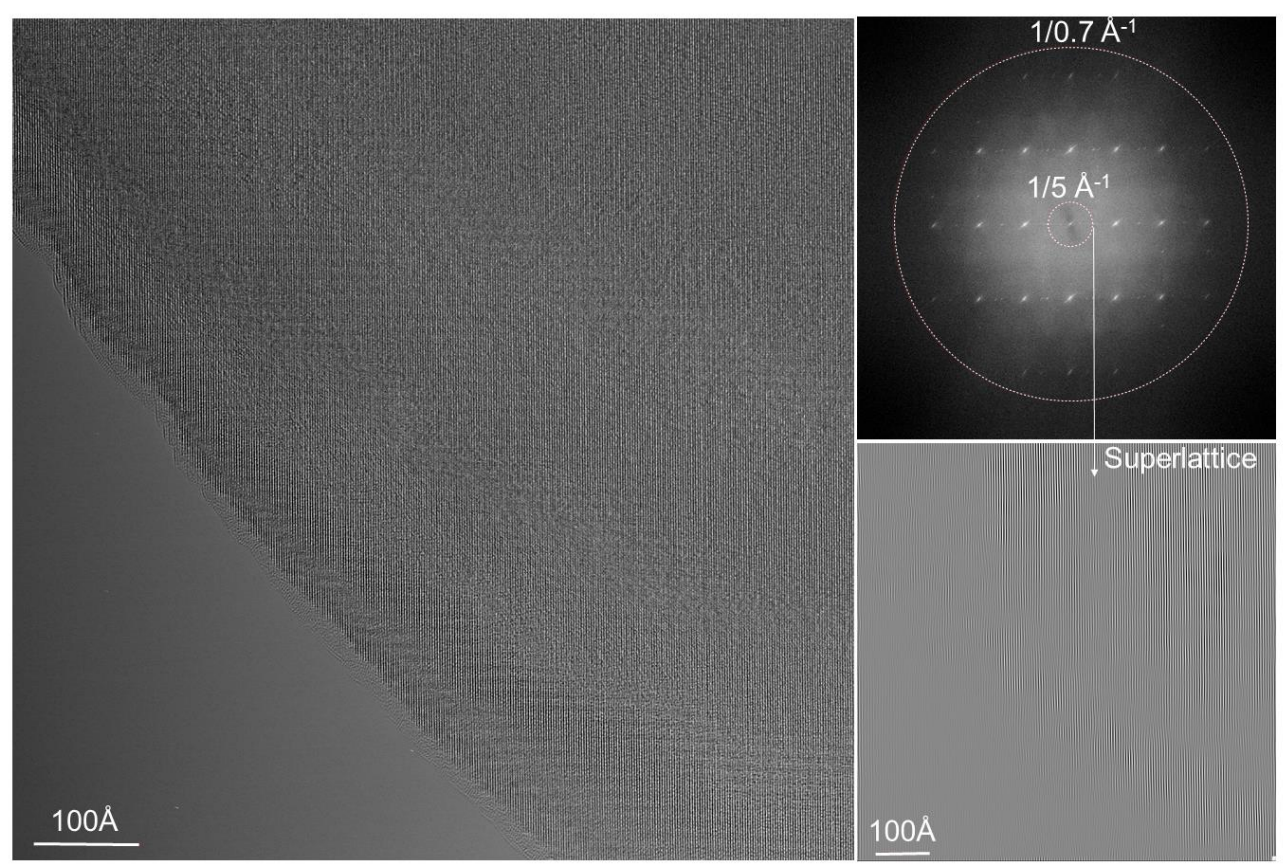

Figure 2. $\mathrm{Al}_{\mathrm{x}} \mathrm{Ga}_{1-\mathrm{x}} \mathrm{N}$ section of a full device structure, imaged at low dose (LD) rates. The image is produced from 60 LD frames, with image alignment. Superlattice reflections which indicate an inhomogeneous distribution of $\mathrm{Ga}$ and $\mathrm{Al}$ are visible (inverse FFT from SL reflections, bottom, right). 


\section{References}

[1] C. Kisielowski, P. Specht, B. Freitag et al., Adv. Funct. Mat. 29, 1807818 (2019)

[2] E.J. VandenBussche; D.J. Flannigan, Nano Letters 19, 6687, 2019

[3] D. Zhang, Y. Zhu, L. Liu et al., Science 359, 675 (2018)

[4] P. Specht, M. Luysberg, J. Chavez et al., Microsc. Microana. 24, S1 (2018)

[5] Work at the Molecular Foundry was supported by the Office of Science, Office of Basic Energy Sciences, of the U.S. Department of Energy under Contract No. DE-AC02-05CH11231.

[6] Project funding by ONR \#N00014-15-1-2714 and DoE NNSA \#PL16-V-GaNPhotodiodePD3Ja is acknowledged. 